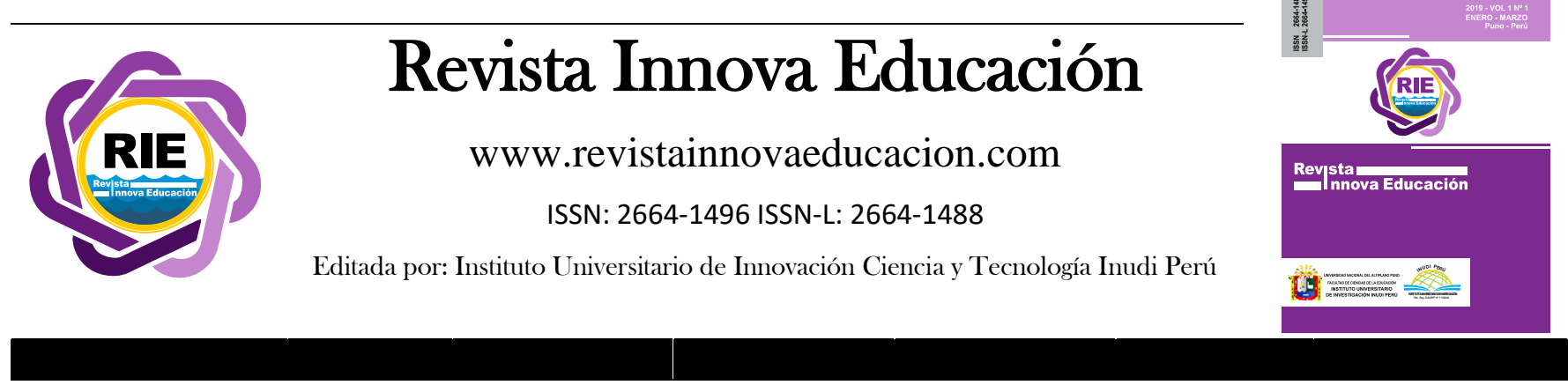

\title{
Programa de aprendizaje de gimnasia básica para niñas del nivel inicial
}

\author{
Learning program of basic gymnastics for girls of the initial level \\ Dometila Mamani $^{1}$ iD; Gino Laque; Nelly Mamani ${ }^{1}$ \\ DOI: https://doi.org/10.35622/j.rie.2019.01.005
}

${ }^{1}$ Universidad Nacional del Altiplano, Perú

Recibido el 21/01/2019/ Aceptado el 27/01/2019

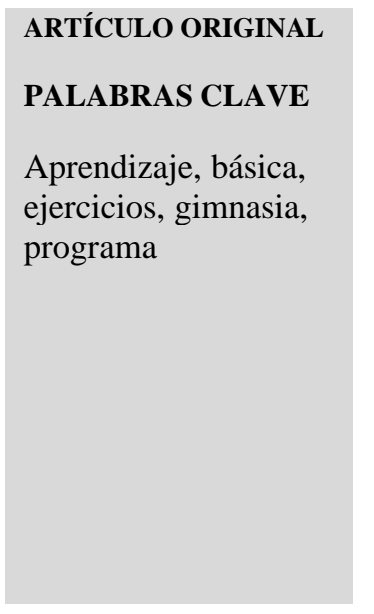

\section{KEYWORDS}

Learning, basic, exercises, gymnastics, program.

\begin{abstract}
La investigación tuvo como objetivo determinar el efecto de la aplicación del Programa DEPREPCEP en el aprendizaje de la gimnasia básica en niñas de la Institución Educativa Inicial Puno 2009. La metodología corresponde a un estudio de nivel experimental de tipo aplicativo, con un diseño de pre y post prueba de un solo grupo. La población de estudio fue de 60 niñas de 5 años y la muestra de 30. Para el análisis de datos se aplicó estadística porcentual y para la comprobación de la hipótesis la prueba estadística $\mathrm{T}$ Student. Los resultados mostraron, antes de la aplicación del programa el $100 \%$ obtuvo un calificativo malo en vertical invertida con apoyo de cabeza, vertical invertida, aspa de molino y arco o araña y $97 \%$ en rodamientos. Después, el $63 \%$ de las niñas obtuvieron calificativo excelente en arco araña, 60\% vertical invertido, $53 \%$ rodamiento corto hacia adelante y hacia atrás y $50 \%$ aspa de molino. Resultados que demuestran diferencia significativa $(\mathrm{p}=0,000)$ entre el nivel de aprendizaje pre y pos test. Por tanto, se concluye que: el Programa DEPREPCEP es efectivo en el aprendizaje de la gimnasia básica en las niñas de educación inicial.
\end{abstract}

The objective of the research was to determine the effect of the application of the DEPREPCEP Program in the learning of basic gymnastics in girls of the Puno 2009 Initial Educational Institution. The methodology corresponds to an experimental-level study of the application type, with a design of pre and post test of a single group. The study population was of 60 girls of 5 years and the sample of 30. For the data analysis, percentage statistics were applied and for the verification of the hypothesis the Student $\mathrm{T}$ statistical test. The results showed, before the application of the program 100\% obtained a bad qualification in inverted vertical with support of head, vertical inverted, blade of mill and bow or spider and $97 \%$ in bearings. Afterwards, $63 \%$ of the girls obtained excellent qualification in spider arch, $60 \%$ inverted vertical, 53\% short forward and backward movement and 50\% windmill blade. Results that show significant difference $(\mathrm{p}=0.000)$ between the level of pre and post test learning. Therefore, it is concluded that: the DEPREPCEP Program is effective in learning basic gymnastics in girls in early childhood education. 


\section{INTRODUCCIÓN}

La Gimnasia a nivel de nuestro país tiene poca acogida para su práctica, y como es de esperar lo propio viene ocurriendo en nuestra Región de Puno; esto indica que la práctica de la Gimnasia es cada vez, menos frecuente. De igual forma la práctica de esta actividad en las Instituciones Educativas de nuestra ciudad es deficiente, debido a que no se incentiva su práctica desde el nivel inicial, por falta de un programa especial sobre la gimnasia básica; considerando que esta etapa es la base para la formación de la gimnasia; al que suma la falta de profesionales capacitados para el desarrollo de este deporte. Un estudio realizado en Puno ha demostrado que el $73,1 \%$ de estudiantes no tienen hábitos para practicar la gimnasia deportiva, ya que no es influida ni incentivada por parte de los docentes por el hecho que no contar con los medios necesarios para el aprendizaje (Ticona, 2017).

La gimnasia general es una modalidad practicada desde tiempos remotos; que fomenta de manera creativa la salud, la integración social y la cultura de la práctica gimnástica en sus diferentes manifestaciones. Es una actividad consistente en ejercicios o movimientos metódicos del cuerpo, que se practica por higiene o por deporte. Esta actividad física es una combinación de ejercicios que exigen del gimnasta unas condiciones físicas excepcionales(Moreno, 2010). Es un deporte en donde se desarrollan las capacidades coordinativas más equilibradas y altamente competitivas desde muy temprana edad (Torres \& Rangel, 2011), porque los ejercicios que se realizan en la Gimnasia incluyen a las acciones elementales, a los ejercicios competitivos, a combinaciones y a todos aquellos que pueden ser divididos o pueden ser continente de alguno de los componentes del sistema desde la competencia, hasta los elementos primarios de valor metodológico(Alfonzo, 2013). En este nivel la influencia de los ejercicios gimnásticos, promueve la enseñanza de los contenidos propios relacionados con el juego, el propio cuerpo, la postura, el movimiento, la expresión, coordinación, lateralidad y la comunicación para favorecer el desarrollo de las nociones perceptivo motoras basadas en la organización y reorganización de las nociones referidas al cuerpo, al espacio, al tiempo y los objetos (Husson, 2008).

Estudios han demostrado que la práctica de la gimnasia favorece al desarrollo corporal de las niñas (Conesa \& Martínez, 2017) ha señalado que la gimnasia además de las trasferencias educativas y de valores que están asociadas a las diferentes modalidades deportivas gimnásticas, potencia la adquisición de hábitos posturales e higiénicos beneficiosos para la salud de los gimnastas. la gimnasia en los niños y niñas aplicada desde tempranas edades les permite tener un aprendizaje significativo y les ayuda a desarrollar mejor sus habilidades motrices, a tener una mejor coordinación, lateralidad y equilibrio (Camacho \& García, 2016).

No obstante, la poca utilización de estrategias metodológicas para el desarrollo de la psicomotricidad hace que el niño y niña no desarrolle sus diversas habilidades y destrezas, cognitivas y motoras mediante la gimnasia (Romero, 2018); Además, los beneficios en los que lo practican ayuda a estimular la coordinación, fuerza, flexibilidad, equilibrio, que busca el acceso a niveles motrices-gimnásticos óptimos (Sánchez, 2014), la aplicación de un Programa de Gimnasia Básica como estrategia para fortalecer la motricidad gruesa, evidencia mejoras notables, donde la parte motriz presentó mayor desempeño porque se fortalecieron la coordinación motora gruesa y fina(Castillo \& Buitrago, 2016). Val Martín \& Zambrano (2017), al proponer la gimnasia cerebral como estrategia para desarrollar la psicomotricidad, recomienda reconocer que los niños y niñas 
de etapa inicial disfrutan de toda actividad física, pues el movimiento es la primera de las artes que ellos manifiestan y el mayor movimiento les permite mantener el control de la inteligencia, situándose en la corteza motora de todo el cerebro; base para el desarrollo físico, intelectual y emocional (Val Martín \& Zambarno, 2017). Además, la gimnasia practicada a través de ciertos movimientos de nuestro cuerpo, estimulamos al cerebro, constituye una alternativa óptima para el desarrollo integral de las capacidades intelectuales, emocionales y creativas de los niños y niñas, ya que su aplicación ayuda a elevar el nivel educativo y cimentar bases sólidas para el futuro(Orellana, 2010) y puede aportar a la formación de la persona (Tapiador, 2014).

Dentro del vasto campo que comprende la Gimnasia Básica constituye el primer peldaño, la base, sobre la que se sustenta la práctica de la cultura física y los deportes; su práctica sistemática permite lograr un nivel elevado de preparación física. Para ello, la enseñanza de habilidades gimnasticas requiere de programas o estrategias de enseñanza; que involucre a los niños en conductas motrices concretas en sus propósitos, siendo necesaria e imprescindible la manipulación intencionada de los ambientes de aprendizaje, mediante la organización de espacios y materiales que promuevan una riqueza vivencial (Gil, Contreras, Gomer, \& Gómez, 2008); mediante "la acción pedagógica que utilice la acción corporal mediante la gimnasia, con el fin de mejorar o normalizar el comportamiento general del niño" (Madrona, Contreras Jordán, Díaz Suárez, \& Navarro, 2006). Al respecto, (Nieto \& Bravo, 2013), diseñó un "Programa de entrenamiento para la escuela de gimnasia artística femenina de la federación deportiva del Azuay para niñas de 5 a 6 años", con la finalidad de mejorar la preparación de las gimnastas respecto al desarrollo físico, biológica y psicológica. Es entonces, que en el estudio se propuso el desarrollo del Programa DREPEPCEP, que significa demostración, ejecución, repetición y perfeccionamiento, corrección de errores y progresión; nace a consecuencia de la insuficiencia y falta de un programa propiamente dicha; por tanto, el estudio tuvo como objetivo determinar el efecto de la aplicación del Programa DEPREPCEP en el nivel de aprendizaje de la Gimnasia Básica en niñas de Educación Inicial de la ciudad de Puno.

\section{MÉTODO Y MATERIALES}

Según el objetivo, el tipo de investigación es experimental de tipo aplicativo que permitió conocer el nivel de aprendizaje de la Gimnasia Básica antes y después de la aplicación del Programa DEPREPCEP. Siendo el diseño preexperimental con un solo grupo.

La población estuvo conformada por 60 niños y niñas de 5 años del II Ciclo de la Institución Educativa Inicial Nro 193 “Club de Leones” Puno y la muestra de 30 niñas obtenidas por el método de Fischer-Arkin-Colton y asignadas al estudio por el método aleatorio simple cuya selección fue por lotería.

En la recolección de datos se aplicó la técnica de la encuesta y como instrumento la lista de cotejo para evaluar el nivel de aprendizaje sobre gimnasia básica. Se aplicó el programa DEPREPCEP, según el plan de experimento y las actividades de aprendizaje. La calificación del instrumento fue en cuatro categorías: Excelente, bueno, regular y malo. El procesamiento de datos se realizó con la estadística descriptiva porcentual y la contrastación de hipótesis con la prueba estadística T-Student. 


\section{RESULTADOS}

Tabla 1 Nivel de aprendizaje de la Gimnasia Básica antes de la aplicación del Programa DEPREPCEP en niñas de Educación Inicial.

\begin{tabular}{|c|c|c|c|c|c|c|c|c|c|c|}
\hline \multirow{3}{*}{$\begin{array}{c}\text { DIMENSIONES DE } \\
\text { LA GIMNASIA } \\
\text { BADICA }\end{array}$} & \multicolumn{8}{|c|}{ PRE TEST } & \multicolumn{2}{|c|}{ TOTAL } \\
\hline & \multicolumn{2}{|c|}{ Malo } & \multicolumn{2}{|c|}{ Regular } & \multicolumn{2}{|c|}{ Bueno } & \multicolumn{2}{|c|}{ Excelente } & \multirow[b]{2}{*}{ Fr. } & \multirow[b]{2}{*}{$\%$} \\
\hline & Fr. & $\%$ & Fr. & $\%$ & Fr. & $\%$ & Fr. & $\%$ & & \\
\hline $\begin{array}{l}\text { Rodamiento corto } \\
\text { hacia adelante }\end{array}$ & 29 & 97 & 1 & 3 & 0 & 0 & 0 & 0 & 30 & 100 \\
\hline $\begin{array}{c}\text { Rodamiento corto } \\
\text { hacia atrás }\end{array}$ & 29 & 97 & 1 & 3 & 0 & 0 & 0 & 0 & 30 & 100 \\
\hline $\begin{array}{c}\text { Vertical invertida con } \\
\text { apoyo de la cabeza }\end{array}$ & 30 & 100 & 0 & 0 & 0 & 0 & 0 & 0 & 30 & 100 \\
\hline Vertical invertida & 30 & 100 & 0 & 0 & 0 & 0 & 0 & 0 & 30 & 100 \\
\hline Aspa de molino & 30 & 100 & 0 & 0 & 0 & 0 & 0 & 0 & 30 & 100 \\
\hline Arco/araña & 30 & 100 & 0 & 0 & 0 & 0 & 0 & 0 & 30 & 100 \\
\hline
\end{tabular}

\section{Fuente: Lista de cotejo}

Los resultados que se presentan en la tabla 1, muestran el nivel de aprendizaje de las niñas de educación inicial sobre la gimnasia básica antes de recibir las enseñanzas del programa DEPREPCEP, donde se observa que el 100\% de las niñas en las dimensiones de vertical invertida con apoyo en cabeza, vertical invertida, aspa de molino y arco araña presentan un aprendizaje malo, así mismo, el $97 \%$ en rodamiento corto hacia adelante y hacia atrás, observándose que ninguna niña tiene un aprendizaje bueno ni excelente.

Tabla 2 Nivel de aprendizaje de la Gimnasia Básica después de la aplicación del Programa DEPREPCEP en niñas de Educación Inicial

\begin{tabular}{|c|c|c|c|c|c|c|c|c|c|c|}
\hline \multirow{3}{*}{$\begin{array}{c}\text { DIMENSIONES DE } \\
\text { LA GIMNASIA } \\
\text { BÁSICA }\end{array}$} & \multicolumn{8}{|c|}{ POST TEST } & \multicolumn{2}{|c|}{ TOTAL } \\
\hline & \multicolumn{2}{|c|}{ Malo } & \multicolumn{2}{|c|}{ Regular } & \multicolumn{2}{|c|}{ Bueno } & \multicolumn{2}{|c|}{ Excelente } & \multirow[b]{2}{*}{ Fr. } & \multirow[b]{2}{*}{$\%$} \\
\hline & Fr. & $\%$ & Fr. & $\%$ & Fr. & $\%$ & Fr. & $\%$ & & \\
\hline $\begin{array}{l}\text { Rodamiento corto hacia } \\
\text { adelante }\end{array}$ & 0 & 0 & 2 & 7 & 12 & 40 & 16 & 53 & 30 & 100 \\
\hline $\begin{array}{c}\text { Rodamiento corto hacia } \\
\text { atrás }\end{array}$ & 0 & 0 & 2 & 7 & 12 & 40 & 16 & 53 & 30 & 100 \\
\hline $\begin{array}{l}\text { Vertical invertida con } \\
\text { apoyo en cabeza }\end{array}$ & 0 & 0 & 2 & 7 & 13 & 43 & 15 & 50 & 30 & 100 \\
\hline Vertical invertida & 0 & 0 & 0 & 0 & 12 & 40 & 18 & 60 & 30 & 100 \\
\hline Aspa de molino & 0 & 0 & 0 & 0 & 14 & 47 & 16 & 53 & 30 & 100 \\
\hline Arco/araña & 0 & 0 & 0 & 0 & 11 & 37 & 19 & 63 & 30 & 100 \\
\hline
\end{tabular}

Fuente: Lista de cotejo 
Los resultados que se presentan en la tabla 2, muestran el nivel de aprendizaje de las niñas de educación inicial sobre la gimnasia básica después de recibir las enseñanzas del programa, donde se observa que el 63\% de las niñas en la dimensión Arco araña obtuvieron un calificativo excelente, también el $60 \%$ en vertical invertida, $53 \%$ en rodamiento corto hacia atrás y adelante y en aspa de molino respectivamente, el 50\% en vertical invertida con apoyo en cabeza, el calificativo bueno se presenta en menor porcentaje y se observa que ninguna niña presenta calificativo malo.

Tabla 3 Diferencia del nivel de aprendizaje de la Gimnasia básica en niñas de Educación Inicial antes y después de la aplicación del Programa DEPREPCEP.

\begin{tabular}{ccccc}
\hline NIVEL & \multicolumn{2}{c}{ PRE TEST } & \multicolumn{2}{c}{ POST TEST } \\
\cline { 2 - 5 } DE APRENDIZAJE & Fr. & \% & Fr. & \% \\
\hline Excelente & 0 & 0 & 16 & 53 \\
Bueno & 0 & 0 & 12 & 40 \\
Regular & 2 & 7 & 2 & 7 \\
Malo & 28 & 93 & 0 & 100 \\
\hline TOTAL & 30 & 100 & 30 &
\end{tabular}

\section{Fuente: Lista de cotejo}

Al describir los resultados del nivel de aprendizaje en la tabla 3, se muestra que las niñas antes de recibir las enseñanzas con el programa DEPREPCEP, el 93\% obtuvo un calificativo malo sobre la Gimnasia Básica y un 2\% calificativo regular; a diferencia después de la aplicación del programa, donde se desarrolló un conjunto de estrategias metodológicas, se logró mejorar el aprendizaje hasta un nivel excelente en el $53 \%$ de las niñas, en el $40 \%$ un calificativo bueno y el $7 \%$ obtuvo un calificativo regular.

\section{DISCUSIÓN}

Los resultados obtenidos sobre el nivel de aprendizaje de la Gimnasia Básica antes de aplicar el Programa DEPREPCEP, presenta resultados desfavorables, porque refleja que niñas tienen un nivel bajo sobre cada una de las dimensiones que comprende el desarrollo de la Gimnasia Básica. El nivel de aprendizaje malo indica que no se conoce los beneficios que otorga la gimnasia básica; este hecho definitivamente hace que niña no desarrolle sus diversas habilidades y destrezas, cognitivas y motoras mediante la gimnasia (Romero, 2018); tampoco, desarrolla o estimula la coordinación, fuerza, flexibilidad, equilibrio, que se necesita para desarrollar los niveles motricesgimnásticos óptimos (Sánchez, 2014); ya que el control postural en condiciones estáticas, es un factor discriminante entre niñas practicantes de gimnasia rítmica (GR) y sus homólogas no deportistas (Guimaraes, Hernández, Rodríguez, \& García, 2015).

Los resultados obtenidos después de aplicar el Programa son muy favorables, las niñas presentan un nivel de aprendizaje excelente en todas las dimensiones de la Gimnasia Básica. Al respecto se menciona que, en tiempos actuales, con la finalidad de mejorar el rendimiento deportivo, se viene creando baterías de test cada vez más específicas para conocer mejor las potencialidades y el desarrollo del deportista en su entorno, así como garantizar una práctica deportiva saludable y propiciar la evaluación de los procesos de entrenamiento(Vernetta, Montosa, 
Beas-Jiménez, \& López-Bedoya, 2017). Basada en la propuesta realizada por los autores, el Programa DEPREPCEP, fue sistematizada y planificada con diversas estrategias metodológicas o la secuencia que permitió aplicar en el proceso de la enseñanza y generar cambios en la estructura cognitiva y procedimental, sobre las diferentes dimensiones que comprende esta actividad, ejecución, que indujo a las niñas a la repetición y perfeccionamiento, con la corrección de errores y progresión que llegaron superar el aprendizaje obtenido; resultados que reflejan la capacidad del programa, gracias al desarrollo de la memoria de forma continua, en sus formas más avanzadas, y a la sistematización y reestructuración del conjunto de relaciones funcionales que comprende el proceso pedagógico (Baquero \& Carretero, 1977).

El nivel de aprendizaje obtenida en forma general demuestra que la sistematización metodológica del proceso de enseñanza en el programa logró mejorar el nivel de aprendizaje, porque fueron capaces de motivar e impulsa la práctica gimnástica en base al aprendizaje obtenido. Vigosky, propone que el aprendizaje además, de disponer habilidades para descubrir por sí mismos, deben ser guiados con explicaciones, demostraciones y hacer posible el aprendizaje cooperativo (Raus, 2017); así como se desarrolló en el programa, donde las niñas fueron guiados para adquirir las habilidades psicomotoras y motivadas para la práctica de la gimnasia básica; contrastando con lo señalado, el programa fue efectivo en el nivel de aprendizaje; existiendo diferencia significativa entre el antes y después de la aplicación del programa; así como se demostró en un estudio experimental realizado con grupos de niños de escuelas deportivas, el Microcircuito aplicado fue eficaz sobre el resto de estrategias y desde el punto de vista de enseñanza (Vernetta, Delgado, \& López, 2010), la utilización de este método resultó ser de gran utilidad en la etapa escolar.

\section{CONCLUSIONES}

El nivel de aprendizaje en las dimensiones rodamiento corto hacia adelante y atrás, vertical invertida con apoyo en cabeza, vertical invertida, aspa de molino y arco araña de la Gimnasia Básica antes de la aplicación del Programa DEPREPCEP es malo.

El nivel de aprendizaje en las dimensiones rodamiento corto hacia adelante y atrás, vertical invertida con apoyo en cabeza, vertical invertida, aspa de molino y arco araña de la Gimnasia Básica después de la aplicación del Programa DEPREPCEP en la mayoría de las niñas es excelente, en cerca de la mitad el nivel de aprendizaje es buena y en menor porcentaje se presenta el aprendizaje regular.

El nivel de aprendizaje sobre la Gimnasia Básica antes de la aplicación del Programa DEPREPCEP es diferente al nivel de aprendizaje obtenido después de recibir las enseñanzas sobre los ejercicios de gimnasia, porque las niñas desde un nivel malo mejoraron su aprendizaje a un nivel excelente y bueno; por tanto, se acepta la hipótesis planteada. 


\section{REFERENCIAS BIBLIOGRÁFICAS}

Alfonzo, A. (2013). El proceso de enseñanza-aprendizaje de la Gimnasia Rítmica en la etapa escolar. Revista Digital Buenos Aires, (180). Retrieved from https://www.efdeportes.com/efd180/ensenanza-aprendizaje-de-la-gimnasia-ritmica.htm

Baquero, R., \& Carretero, M. (1977). Vigotsky y el aprendizaje escolar. (Ayque Grupo, Ed.) (Segunda). Argentina. Retrieved from http://www.terras.edu.ar/biblioteca /6/6PE_Baquero_2_Unidad_2.pdf

Camacho, V., \& García, M. . (2016). La gimnasia cerebral y la coordinación motora fina de los niños y niñas del subnivel ii de educación inicial de la Unidad Educativa Juan Montalvo del Cantón Ambato. Ecuador. Retrieved from http://repositorio.uta.edu.ec /bitstream/123456789/24283/1/Paucar Camacho Vilma Beatriz.pdf

Castillo, L., \& Buitrago, C. (2016). La Gimnasia básica como estrategia para fortalecer la motricidad gruesa en niños y niñas de 7 a 8 años de edad. Colombia. Retrieved from https://repositorio.uptc.edu.co/bitstream/001/1939/1/TGT-464.pdf

Conesa, E., \& Martínez, F. (2017). Una modalidad saludable de Gimnasia en edad escolar La gimnasia estética de grupo. E-Balonmano.Com: Revista de Ciencias Del Deporte, 13(1). Retrieved from https://www.redalyc.org/html/865/86551349004/

Gil, P., Contreras, O., Gomer, S., \& Gómez, B. (2008). Justificación de la educación física en la educación infantil. Educación y Educadores, 11(2), 19. Retrieved from http://www.scielo.org.co/pdf/eded/v11n2/v11n2a10.pdf

Guimaraes, D., Hernández, M., Rodríguez, D., \& García, J. M. (2015). Efecto del entrenamiento sistemático de gimnasia rítmica sobre el control postural de niñas adolescentes. Revista Andaluza de Medicina Del Deporte, 8(2), 54-60. https://doi.org/10.1016/j.ramd.2014.11.001

Husson, M. (2008). La gimnasia como contenido educativo. Retrieved from http://www.memoria.fahce.unlp.edu.ar/trab_eventos/ev.685/ev.685.pdf

Madrona, P. G., Contreras Jordán, O. R., Díaz Suárez, A., \& Navarro, Á. L. (2006). La educación física en su contribución al proceso formativo de la educación infantil. Revista de Educación (Vol. 339). Retrieved from http://www.revistaeducacion.mec.es/re339/re339a18.pdf

Moreno, P. (2010). Unidad Didáctica Educación Física. Progresión pedagógica aprendizaje de rondada flic flac en Educación Secundaria. Revista Digital, 15(147). Retrieved from https://www.efdeportes.com/efd147/ 
Nieto, Y., \& Bravo, L. (2013). Programa de entrenamiento para la escuela de gimnasia artística femenina de la federación deportiva del Azuay de 5 a 6 años. Ecuador. Retrieved from https://dspace.ups.edu.ec/bitstream/123456789/6437/1/UPS-CT003068.pdf

Orellana, D. (2010). Tesina previa a la obtención del título de licenciada en psicología en la especialidad de educación temprana. Ecuador. Retrieved from http://dspace.ucuenca.edu.ec/bitstream/123456789/2183/1/tps687.pdf

Raus, M. (2017). Vygotsky.Principios y conceptos básicos de la teoría del Constructivismo Social. Retrieved January 11, 2019, from https://educacionparalasolidaridad.com /2017/01/18/vygotsky-principios-y-conceptos-basicos-de-la-teoria-del-constructivismosocial/

Romero, Z. (2018). Desarrollo de la psicomotricidad en los estudiantes del nivel inicial de la institución educativa $\mathrm{N}^{\circ}$ 429-36 Ayahuanco de Huanta región Ayacucho en el año académico 2018. Universidad Católica Los Ángeles de Chimbote. Retrieved from http://repositorio.uladech.edu.pe/handle/123456789/4850?show=full

Sánchez, B. (2014). Manual de gimnasia básica para el desarrollo de las capacidadeas emocinales y coordinativas en niños con sindrome de Down de 9 a 11 años en edad escolar de la Fundación FASINARM. Retrieved from http://repositorio.ug.edu.ec/handle/redug/19216

Tapiador, M. (2014). Recordando los valores de la gimnasia artística femenina. Apunts Educación Fisica I Esports, (64). Retrieved from http://www.revista-apunts.com/es/hemeroteca ?article $=460$

Ticona, W. (2017). Factores que influyen en la enseñanza aprendizaje de la gimnasia deportiva en las Instituciones Educativas primarias de la Ciudad de Puno - 2015. Retrieved January 13, 2019, from https://alicia.concytec.gob.pe/vufind/Record/RNAP_1fb40fa1d2a602b99a48eea

Torres, R., \& Rangel, B. (2011). Entrenamiento de gimnasia, crecimiento y madurez biológica en gimnastas prepuberales y puberales. Repositorio Académico, 7(7), 107-110. Retrieved from http://eprints.uanl.mx/5474/

Val Martín, P., \& Zambarno, T. (2017). La gimnasia cerebral como estrategia para desarrollar la psicomotricidad en los niños y niñas. Lecturas: Educación Física y Deportes, 22(235). Retrieved from https://efdeportes.com/index.php/EFDeportes/article/view/42/6

Vernetta, M., Delgado, M. ., \& López, J. (2010). Aprendizaje en gimnasia artística. Un estudio experimental con niños que analiza ciertas variables del proceso. European Journal of Human 
Movement, 2(0), 93-112. Retrieved from https://recyt.fecyt.es/index.php/ejhm/ article/view/56118/33928

Vernetta, M., Montosa, I., Beas-Jiménez, J., \& López-Bedoya, J. (2017). Batería Funcional ARISTO en Gimnasia Rítmica: protocolo de test específicos para la evaluación de jóvenes gimnastas en un ámbito de entrenamiento saludable. Revista Andaluza de Medicina del Deporte, 10(3), 112-119. https://doi.org/10.1016/J.RAMD.2017.02.001 